\title{
Backscattering Between Helical Edge States via Dynamic Nuclear Polarization
}

\author{
Adrian Del Maestro, ${ }^{1}$ Timo Hyart, ${ }^{2}$ and Bernd Rosenow ${ }^{2}$ \\ ${ }^{1}$ Department of Physics, University of Vermont, Burlington, VT 05405, USA \\ ${ }^{2}$ Institut für Theoretische Physik, Universität Leipzig, D-04103, Leipzig, Germany
}

(Dated: January 12, 2021)

\begin{abstract}
We show that that the non-equilibrium spin polarization of one dimensional helical edge states at the boundary of a two dimensional topological insulator can dynamically induce a polarization of nuclei via the hyperfine interaction. When combined with a spatially inhomogeneous Rashba coupling, the steady state polarization of the nuclei produces backscattering between the topologically protected edge states leading to a reduction in the conductance which persists to zero temperature. We study these effects in both short and long edges, uncovering deviations from Ohmic transport at finite temperature and a current noise spectrum which may hold the fingerprints for experimental verification of the backscattering mechanism.
\end{abstract}

PACS numbers: 72.15.Lh, 72.25.-b, 85.75.-d, 31.30.Gs

Topological insulators have a bulk band gap, but cannot be adiabatically connected to the conventional insulators without closing the energy gap [1-4] because they are characterized by a non-trivial topological number. The quantum spin Hall (QSH) insulators belong to the class of two dimensional time reversal (TR) invariant $\mathbb{Z}_{2}$ topological insulators. They support a pair of gapless helical edge states, which give rise to unique, experimentally confirmed [5] 8] electrical properties. Due to their helical nature, QSH edge states are protected against elastic back-scattering from TR symmetric perturbations: there is destructive interference between two time reversed amplitudes, one where spin is rotated in a, say, clockwise manner and a second one with anti-clockwise rotation. In the interference term, this amounts to a full rotation of a spin- $1 / 2$, corresponding to a factor of minus one, which cancels the contribution of the direct term. Backscattering due to TR breaking perturbations $9-12$ and from inelastic processes 17, 13, 18 is however allowed.

The protection of the edge states in the presence of TR symmetry can be exemplified by considering the scattering due to a spatially random Rashba spin-orbit coupling [19 22. In Fourier space, the matrix element for this process includes a factor $\left(k+k^{\prime}\right)$, where $k$ and $k^{\prime}$ are the momenta of initial and final states, respectively. For a TR invariant Hamiltonian, the dispersion relation satisfies $\epsilon_{k}=\epsilon_{-k}$, such that energy conservation enforces $k^{\prime}=-k$ implying a vanishing matrix element for backscattering. In the presence of an in-plane magnetic field however, there is a Zeeman splitting $2 \Delta=g \mu_{\mathrm{B}} B$ between opposite spin directions. For a linearized dispersion $\epsilon(k)=\hbar v k$ this gives rise to a non-vanishing prefactor $\left(k+k^{\prime}\right)=2 \Delta / \hbar v$ and yields a finite mean free path $\ell \propto 1 / \Delta^{2}$. This mechanism can explain the experimentally observed dependence of the quantum spin Hall edge conductance on an in-plane magnetic field [5, 6].

In this letter, we discuss the influence of dynamically polarized nuclear spins on the conductance of QSH edge channels depicted in Fig. 1. We argue that flip-flop scat-

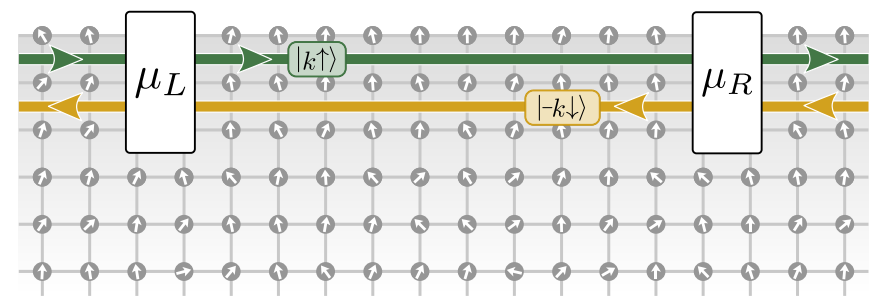

FIG. 1. Quantum spin Hall edge states dynamically polarize nuclear spins via a hyperfine interaction. Although the edge states are drawn as sharp lines, their wavefunctions may overlap a large number of nuclei. Only one edge is shown, but there is an equivalent and independent transport channel at the opposite edge, where the spin polarizations are reversed.

tering between electronic and nuclear spins creates a dynamic nuclear polarization, which has the same effect as an external Zeeman field and gives rise to backscattering between helical edge states. The magnitude of the nuclear spin polarization is determined by the ratio of bias voltage and temperature. Thus, for a long edge of length $L \gg \ell$, the local temperature profile governed by a balance between Joule heating and electronic heat transport determines the conductance. We find a non-linear current-voltage characteristic at finite temperature and a peculiar relation between applied voltage and noise power, which together with hysteresis in the currentvoltage characteristic are signatures of backscattering due to a dynamic nuclear polarization. Importantly, this mechanism stays effective in the low-temperature limit, similar to spin-flip scattering in $\nu=2$ quantum Hall edges [23].

At low energies in the presence of a nuclear spin polarization, the helical edge electrons have an effective Dirac spectrum governed by

$$
H_{0}=\int d x \psi_{\alpha}^{\dagger}(x) \sigma_{\alpha \beta}^{z}\left(-i v \hbar \partial_{x}+\frac{1}{2} A\langle I\rangle\right) \psi_{\beta}(x),
$$


where $\psi_{\alpha=\uparrow, \downarrow}^{\dagger}(x)$ is a fermionic field corresponding to a right moving (spin-up) or left moving (spin-down) electron with Fermi velocity $v, \sigma^{z}$ is a Pauli matrix in spin space, and repeated indices are summed over. The polarization of nuclear spins is generated via the hyperfine dynamic Hamiltonian [24]

$$
H_{\text {flip }}=\frac{A}{2 \rho_{n}} \sum_{i} \psi_{\alpha}^{\dagger}\left(x_{i}\right)\left(\sigma_{\alpha \beta}^{+} I_{i}^{-}+\sigma_{\alpha \beta}^{-} I_{i}^{+}\right) \psi_{\beta}\left(x_{i}\right)
$$

where $\boldsymbol{I}_{i}$ is the spin operator for a nucleus located at position $\boldsymbol{r}_{i}=\left(x_{i}, y_{i}, z_{i}\right), \sigma^{ \pm}=\left(\sigma_{x} \pm i \sigma_{y}\right) / 2$, and $\rho_{n}$ is the effective one dimensional density of nuclei seen by the edge electrons. Spin flips are generated by this effective contact term, where the strength of the hyperfine interaction, $A$, can be measured using optical or electron paramagnetic resonance experiments 25. Backscattering between right movers and left movers can be induced by a spatially dependent Rashba spin-orbit coupling term that can arise as a result of electrostatic potential disorder 1922$]$. The interactions are described by

$$
H_{R}=\int d x \psi_{\alpha}^{\dagger}(x) \sigma_{\alpha \beta}^{y}\left\{a(x), i \partial_{x}\right\} \psi_{\beta}(x)
$$

where the strength of the inhomogeneous coupling is set by the scale of its local correlations: $\left\langle a(x) a\left(x^{\prime}\right)\right\rangle=$ $V_{R} \delta\left(x-x^{\prime}\right)$. The total Hamiltonian is given by $H=$ $H_{0}+H_{\text {flip }}+H_{\mathrm{R}}$ where the effective Zeeman gap originating from the polarized nuclei $A\langle I\rangle$ will need to be determined from the steady state of nuclear polarization.

To this end, we begin by calculating the net scattering rate per unit length, $\Gamma$, describing the transfer of electrons between the right and left moving spin polarized edge states, $|k \uparrow\rangle$ and $\left|-k^{\prime} \downarrow\right\rangle$, due to the interaction between the electron and the nuclear spins $H_{\text {flip }}$. Employing Fermi's golden rule we obtain $26-28$

$$
\begin{gathered}
\Gamma=\frac{A^{2}}{8 \pi \hbar^{3} v^{2} \rho_{n}} \int d \epsilon\left\{(1 / 2-\langle I(t)\rangle) f_{+}(\epsilon)\left[1-f_{-}(\epsilon)\right]\right. \\
\left.-(1 / 2+\langle I(t)\rangle) f_{-}(\epsilon)\left[1-f_{+}(\epsilon)\right]\right\}
\end{gathered}
$$

where $f_{ \pm}(\epsilon)$ are the distribution functions for the right and left moving edge states, respectively.

Because each scattering event is associated with a nuclear spin flip, the time evolution of the nuclear spin polarization can be described with a rate equation $\partial_{t}\langle I(t)\rangle=\Gamma / \rho_{n}-\Gamma_{d}\langle I(t)\rangle / \rho_{n}$, where $\Gamma_{d}$ is a phenomenological nuclear spin relaxation rate encompassing all other mechanisms that lead to nuclear spin flips. In quantum Hall systems, the nuclear spin relaxation rate is typically much smaller than the spin flip scattering rate that originates from the dipole interaction between the nuclear spins and the spin polarized edge states 23. Due to their similarity with the QSH edge states considered here, we expect that $\Gamma_{d} \ll \Gamma$.
The rate equation for the nuclear spin polarization can be solved for arbitrary electron distribution functions $f_{ \pm}(\epsilon)$. Assuming that the nuclear spin polarization is initially zero, the time evolution is described by $\langle I(t)\rangle=\langle I\rangle\left(1-e^{-t / \tau_{n}}\right)$, where

$$
\begin{aligned}
\langle I\rangle & =\frac{\int d \epsilon\left[f_{+}(\epsilon)-f_{-}(\epsilon)\right] / 2}{\int d \epsilon\left\{f_{+}(\epsilon)+f_{-}(\epsilon)\left[1-2 f_{+}(\epsilon)\right]\right\}+\frac{\Gamma_{d} 8 \pi \hbar^{3} v^{2} \rho_{n}}{A^{2}}}, \\
\tau_{n}^{-1} & =\frac{A^{2} \int d \epsilon\left\{f_{+}(\epsilon)+f_{-}(\epsilon)\left[1-2 f_{+}(\epsilon)\right]\right\}}{8 \pi \hbar^{3} v^{2} \rho_{n}^{2}}+\frac{\Gamma_{d}}{\rho_{n}} .
\end{aligned}
$$

It is difficult to obtain a reliable estimate for the characteristic time-scale $\tau_{n}$, where the steady-state nuclear polarization $\langle I\rangle$ is achieved. Based on the experiments in the quantum Hall systems [23], we expect that the first term in $\tau_{n}^{-1}$ dominates and $\tau_{n}$ is on the order of tens or hundreds of seconds.

To evaluate the effect of a finite nuclear polarization $\langle I\rangle$ on electrical transport, we first concentrate on the case of a short edge, where the distance between contacts $L$ is much shorter than the mean free path associated with the spin flip scattering. The distribution functions are then determined by the Fermi functions $f_{ \pm}(\epsilon)=$ $f_{0}\left(\epsilon-\mu_{L / R}, T\right)$, where $f_{0}(\epsilon, T)=\left[1+\exp \left(\epsilon / k_{\mathrm{B}} T\right)\right]^{-1}$, and $\mu_{L / R}$ are the chemical potentials of the left/right reservoirs, respectively. At zero temperature $\langle I\rangle=$ $\frac{1}{2}\left[1+\frac{\Gamma_{d} 8 \pi \hbar^{3} v^{2} \rho_{n}}{A^{2}\left(\mu_{L}-\mu_{R}\right)}\right]^{-1}$, so that in the absence of relaxation, the nuclear spins become fully polarized. For $\Gamma_{d}=0$, the temperature dependence of the nuclear spin polarization is described by $\langle I\rangle=\frac{1}{2} \tanh \left[\left(\mu_{L}-\mu_{R}\right) / 2 k_{\mathrm{B}} T\right]$.

We now turn to evaluating the backscattering rate due to the random Rashba spin orbit coupling in the presence of a dynamically generated nuclear spin polarization $\langle I\rangle$. Denoting initial and final electronic states by their momenta $|k \uparrow\rangle$ and $\left|k^{\prime} \downarrow\right\rangle$, we find the matrix element $\left\langle k^{\prime}\left|H_{R}\right| k\right\rangle=-i\left(k+k^{\prime}\right) \hat{a}\left(k^{\prime}-k\right) / L$. Taking into account that due to energy conservation $\left(k+k^{\prime}\right)=-A\langle I\rangle / v \hbar$, we find for the disorder averaged scattering rate $W_{k k^{\prime}}=$ $2 \pi V_{R} A^{2}\langle I\rangle^{2} \delta\left(\epsilon_{k}-\epsilon_{k^{\prime}}\right) /\left(\hbar^{3} v^{2} L\right)$. The total Rashba scattering rate is then

$$
\frac{1}{\tau_{R}} \equiv \sum_{k^{\prime}} W_{k k^{\prime}}=A^{2}\langle I\rangle^{2} \frac{V_{R}}{\hbar^{4} v^{3}}
$$

The edge conductivity due to Rashba scattering is $\sigma=$ $\left(e^{2} / \pi \hbar\right) \ell$ with $\ell=v \tau_{R} / 2$ (the extra factor $1 / 2$ accounts for the difference between single particle and transport relaxation time). It is related to the two-terminal conductance via

$$
G=\left(\frac{h}{e^{2}}+\frac{L}{\sigma}\right)^{-1}
$$

In the limit of a short edge with $L \ll \ell$, the conductance is changed by

$$
\delta G=-\frac{e^{2}}{h} A^{2}\langle I\rangle^{2} \frac{V_{R}}{\hbar^{4} v^{4}} L
$$




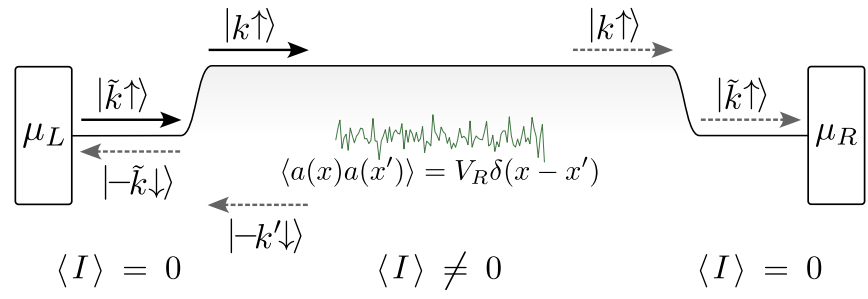

FIG. 2. An outline of sequential scattering processes for the helical edge states. It is assumed that the nuclei are unpolarized $(\langle I\rangle=0)$ in the leads but are dynamically polarized $(\langle I\rangle \neq 0)$ between them in a region with potential disorder giving rise to random Rashba scattering.

This equation can be directly verified by calculating the change of current caused by scattering between the spin polarized edge states. Here, we assume that the phase space for scattering is not changed by the presence of a spin splitting due to the nuclear polarization. This can be understood by calculating scattering states from the time independent Schröedinger equation describing energy eigenstates as pictured schematically in Fig. 2. We assume that there is no spin splitting in the reservoirs, and that it is switched on and off in regions of the edge where there is no Rashba scattering. Since the nuclear magnetization only couples to the electron spin, which is assumed to be a good quantum number, a finite $\langle I\rangle$ leads to a change in wave vector, such that Rashba scattering has a finite matrix element. After the scattering, $\langle I\rangle$ is switched off again before entering the reservoir, so the initial and final momentum are equal in magnitude.

For long edges with $L \gg \ell$, the distribution functions of the electrons are no longer determined by the reservoir distributions but have to be calculated from a solution of a kinetic equation. In the following, we assume that the Coulomb interaction between counter-propagating edge states gives rise to an inelastic mean free path approximately equal to the elastic one [29. Thus, it seems reasonable to assume that a long edge region can be described locally by equilibrium distributions with position dependent chemical potential $\mu(x)$, temperature $T(x)$, and nuclear polarization $\langle I\rangle(x)$. Neglecting the relaxation of nuclear spins, the nuclear polarization is related to the difference in chemical potential between left and right movers, $\delta \mu$, which in turn is determined by the electrical current $j$ via $\delta \mu=j h / e$. As current is conserved along the edge, we have

$$
\langle I\rangle(x)=\frac{1}{2} \tanh \left(\frac{j \pi \hbar}{e k_{\mathrm{B}} T(x)}\right) .
$$

Expressing the electron distribution function as

$$
f(k, x)=f_{0}\left(\xi_{k}, T(x)\right)+\delta f(k, x),
$$

with $\xi_{k}=\epsilon_{k}+\operatorname{sign}(k) A\langle I\rangle(x)-\mu(x)$ subject to the linearized steady state Boltzmann equation $v_{k} \partial_{x} f_{0}=$ $-\delta f 2 / \tau_{R}$, we find the solution

$$
\delta f(k, x)=\frac{\tau_{R}}{2} \delta\left(\xi_{k}\right)\left[\operatorname{sign}(k) A \partial_{x}\langle I\rangle-\partial_{x} \mu-\frac{\xi_{k}}{T^{2}} \partial_{x} T\right] .
$$

The current is obtained as $j=e \int \frac{d k}{2 \pi} v_{k} \delta f$, giving rise to a position dependent conductivity

$$
\sigma(x)=\frac{e^{2}}{\pi \hbar} \frac{\hbar^{4} v^{4}}{2 V_{R}} \frac{1}{A^{2}\langle I\rangle^{2}(x)} .
$$

The conductivity depends on temperature via the expectation value of the nuclear polarization in Eq. (8). In principle, interaction effects give rise to an additional, explicit temperature dependence of the backscattering rate, which is not included in the following. In order to determine the conductance, knowledge of the temperature profile $T(x)$ along the edge is needed. In the limit of small bias, the edge temperature is given by the bath temperature, $T(x) \equiv T$. Linearizing Eq. (8) we obtain

$$
j^{3}=\frac{e}{\pi \hbar} \frac{2 \hbar^{4} v^{4}}{V_{R}}\left(\frac{e k_{\mathrm{B}} T}{A \pi \hbar}\right)^{2}\left(-\frac{\partial \mu}{\partial x}\right),
$$

a nonlinear current voltage relation, where in the limit $\sigma / L \ll e^{2} / h$ one finds $j \propto T^{2 / 3} \mu^{1 / 3}$.

In general, the temperature distribution $T(x)$ must be calculated self-consistently, taking into account Joule heating due to the transport current [30, 31]. Denoting the heat current by $j_{Q}$, it is related to the local temperature gradient by the heat conductivity $\kappa=$ $\sigma T\left(k_{\mathrm{B}} / e\right)^{2}\left(\pi^{2} / 3\right)$ as $j_{Q}=-\kappa \partial_{x} T$. Joule heating determines the divergence of the heat current according to $\partial_{x} j_{Q}=j^{2} / \sigma$, such that

$$
-\sigma(x) \partial_{x}\left[\sigma(x) \partial_{x} T^{2}(x)\right]=j^{2}\left(\frac{e}{k_{\mathrm{B}}}\right)^{2} \frac{6}{\pi^{2}} .
$$

Together with Eqs. (8) and (11) this constitutes a nonlinear differential equation for the temperature profile. We now discuss the solution of Eq. (13) in the limit where the bath temperature can be neglected as compared to the temperature generated by Joule heating, e.g. we use the boundary conditions $T(0)=T(L)=0$. Introducing the length scale $\ell_{0}=(\hbar v)^{4} /\left(2 V_{R} A^{2}\right)$, we define a dimensionless coordinate $\bar{x} \equiv x / \ell_{0}$ and temperature variable $t(\bar{x}) \equiv T(x) / \Theta(j)$ with $\Theta(j)=j \pi \hbar / e k_{\mathrm{B}}$. Eq. (13) may now be numerically integrated yielding the dimensionless temperature profile and nuclear polarization shown in Fig. 3 for fixed $L / \ell_{0} \gg 1$.

The chemical potential difference $\Delta \mu$ between the left and right reservoirs is related to the current via

$$
\Delta \mu=j \frac{\pi \hbar}{4 e} \int_{0}^{L / \ell_{0}} d \bar{x} \tanh ^{2}\left(\frac{1}{t(\bar{x})}\right) .
$$

Since the functional form of the rescaled temperature profile $t(\bar{x})$ only depends on the dimensionless parameter $L / \ell_{0}$ through the boundary conditions of Eq. 13, 


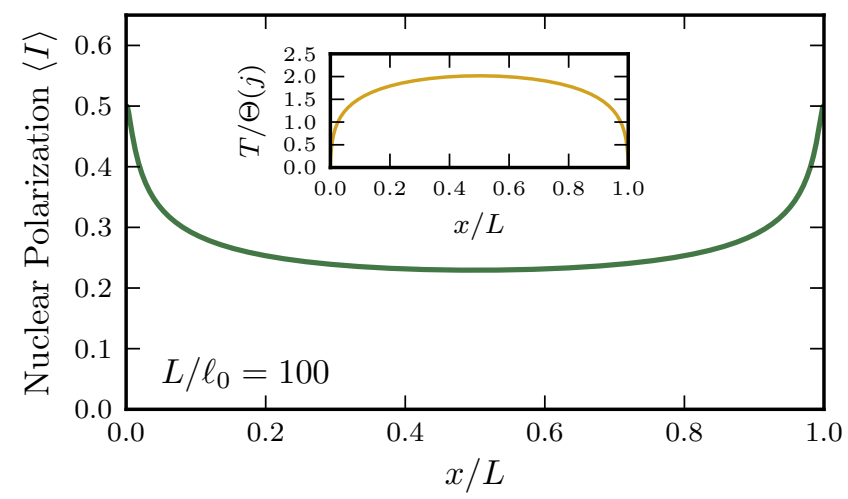

FIG. 3. The induced dynamic nuclear polarization $\langle I\rangle(x)$ computed from Eq. (8) for a long $\left(L / \ell_{0} \gg 1\right)$ edge using the dimensionless temperature profile shown in the inset.

it is clear from Eq. (14) that the resistance of a long edge is Ohmic in the limit of zero bath temperature. However, we obtain an interesting scaling $\pi \hbar G / e^{2} \propto\left(l_{0} / L\right)^{0.35}$ of the conductance with the length of the wire. The current noise can be obtained from [30, 31.

$$
S=4 \frac{1}{L^{2}} \int_{0}^{L} d x \sigma(x) k_{\mathrm{B}} T(x)
$$

and can be expressed in the form $S=F G V$, where $F$ denotes the Fano factor and $G$ is the conductance obtained from the current voltage relation Eq. (14). By studying the behavior of Eq. (15) in the long edge limit, as shown in Fig. 4, we extract a numerical result for the Fano factor of $F=1.219(2)$ where the number in the bracket indicates the uncertainty in the final digit. By comparison, the Fano factor for a one dimensional diffusive wire much longer than the inelastic mean free path is $\sqrt{3} / 2$ [32, 33], if cooling due to electron-phonon coupling can be neglected.

Before attempting to apply the results discussed here to the HgTe quantum wells studied in Ref. [5] it is useful to put them in the context of other potential backscattering mechanisms that have been proposed to explain the observed deviation from ballistic edge transport. In the presence of both time reversal symmetry breaking and disorder, the helical edge states will be localized, as studied in the presence of magnetic fields and impurities [9], random magnetic fluxes [10] and magnetic impurities [11, 12. Alternatively, back-scattering becomes possible in the presence of dephasing processes, which have been simulated phenomenologically using Büttiker's virtual probes [7, 13]. Budich et al. [14] found that inelastic processes due to electron-phonon interactions in the presence of spin orbit coupling cannot cause backscattering between helical edge states to leading order. Other studies have shown that avenues towards backscattering from magnetic impurities alone [15], electron-electron interac-

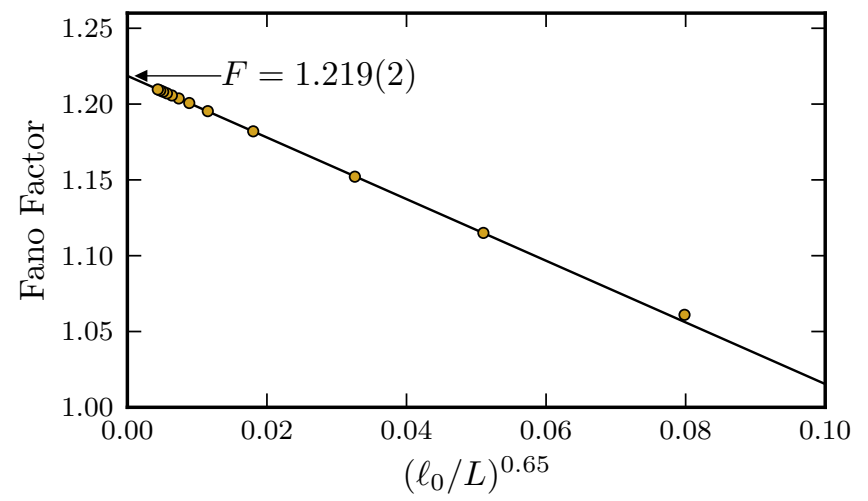

FIG. 4. The Fano factor calculated from Eq. 15 is found to be parametrized by the finite size scaling form $\bar{F}=1.219(2)-$ $2.03(3)\left(\ell_{0} / L\right)^{0.65(3)}$.

tions [16, 17] or the loss of axial spin symmetry [18] are all suppressed in the low temperature limit.

Backscattering from dynamic nuclear polarization persists to zero temperature. In order to obtain an estimate for the mean free path $\ell_{0}$, we exploit the interpretation of $A\langle I\rangle$ as a local effective Zeeman splitting. In this way, we can determine the parameters in the scattering rate by comparison with the effects of backscattering due to an in-plane magnetic field in the presence of a spatially random Rashba coupling. Examining Ref. [5] we estimate that $(\hbar v)^{4} / 2 V_{R} \approx 0.14(\mu \mathrm{eV})^{2} \mu \mathrm{m}$, which in combination with a hyperfine constant of $A \approx 60 \mu \mathrm{eV}$ for interactions of electrons with the fully polarized nuclei of mercury [34] produces $\ell_{0} \approx 40 \mu \mathrm{m}$.

A.D. acknowledges financial support from the University of Vermont and the gracious hospitality of the Institut für Theoretische Physik, Universität Leipzig where the majority of this work was completed.

[1] C. L. Kane and E. J. Mele, Phys. Rev. Lett. 95, 226801 (2005).

[2] B. A. Bernevig, T. L. Hughes, and S. C. Zhang, Science 314, 1757 (2006).

[3] M. Z. Hasan and C. L. Kane, Rev. Mod. Phys. 82, 3045 (2010)

[4] X.-L. Qi and S.-C. Zhang, Rev. Mod. Phys. 83, 1057 (2011)

[5] M. König, S. Wiedmann, C. Brune, A. Roth, H. Buhmann, L. W. Molenkamp, X. L. Qi, and S. C. Zhang, Science 318, 766 (2007).

[6] M. König, H. Buhmann, L. W. Molenkamp, T. Hughes, C.-X. Liu, X.-L. Qi, and S.-C. Zhang, J. Phys. Soc. Jpn. 77, 031007 (2008).

[7] A. Roth, C. Brune, H. Buhmann, L. W. Molenkamp, J. Maciejko, X. L. Qi, and S. C. Zhang, Science 325, 294 (2009). 
[8] C. Brüne, A. Roth, H. Buhmann, E. M. Hankiewicz, L. W. Molenkamp, J. Maciejko, X.-L. Qi, and S.-C. Zhang, Nature Phys. 8, 486 (2012).

[9] J. Maciejko, X.-L. Qi, and S.-C. Zhang, Phys. Rev. B 82, $155310(2010)$.

[10] P. Delplace, J. Li, and M. Büttiker, arXiv (2012), arXiv:1207.2400 [cond-mat],

[11] Y. Tanaka, A. Furusaki, and K. A. Matveev, Phys. Rev. Lett. 106, 236402 (2011).

[12] K. Hattori, J. Phys. Soc. Jpn. 80, 124712 (2011).

[13] H. Jiang, S. Cheng, Q. Sun, and X. C. Xie, Phys. Rev. Lett. 103, 36803 (2009).

[14] J. C. Budich, F. Dolcini, P. Recher, and B. Trauzettel, Phys. Rev. Lett. 108 (2012).

[15] J. Maciejko, C. Liu, Y. Oreg, X.-L. Qi, C. Wu, and S.-C. Zhang, Phys. Rev. Lett. 102, 256803 (2009).

[16] F. Crépin, J. C. Budich, F. Dolcini, P. Recher, and B. Trauzettel, arXiv (2012), arXiv:1205.0374 [cond-mat].

[17] N. Lezmy, Y. Oreg, and M. Berkooz, Phys. Rev. B 85, 235304 (2012)

[18] T. Schmidt, S. Rachel, F. von Oppen, and L. Glazman, Phys. Rev. Lett. 108, 156402 (2012).

[19] E. Y. Sherman, Phys. Rev. B 67, 161303 (2003).

[20] L. Golub and E. Ivchenko, Phys. Rev. B 69, 115333 (2004).
[21] A. Strom, H. Johannesson, and G. I. Japaridze, Phys. Rev. Lett. 104, 256804 (2010).

[22] D. G. Rothe, R. W. Reinthaler, C.-X. Liu, L. W. Molenkamp, S. C. Zhang, and E. M. Hankiewicz, New J. Phys. 12, 065012 (2010).

[23] K. Wald, L. Kouwenhoven, P. McEuen, N. van der Vaart, and C. Foxon, Phys. Rev. Lett. 73, 1011 (1994).

[24] C. Slichter, Principles of Magnetic Resonance, Springer Series in Solid-State Sciences (Springer, Berlin, 1990).

[25] N. J. Stone, Atom. Data Nucl. Data. 90, 75 (2005)

[26] A. Overhauser, Phys. Rev. 89, 689 (1953).

[27] V. Tripathi, A. C. H. Cheung, and N. R. Cooper, Europhys Lett. 81, 68001 (2008).

[28] A. Lunde and G. Platero, Phys. Rev. B 86, 035112 (2012).

[29] D. Bagrets, I. Gornyi, and D. Polyakov, Phys. Rev. B 80, 113403 (2009).

[30] A. H. Steinbach, J. M. Martinis, and M. H. Devoret, Phys. Rev. Lett. 76, 3806 (1996).

[31] F. von Oppen, Phys. Rev. B 56, 9674 (1997).

[32] K. E. Nagaev, Phys. Rev. B 52, 4740 (1995).

[33] V. I. V. I. Kozub and A. M. Rudin, Phys. Rev. B 52, 7853 (1995).

[34] G. S. Bogle, J. N. Dodd, and W. L. McLean, Proc. Phys. Soc. B 70, 796 (2002). 